RESEARCH HIGHLIGHT

\title{
Break the net, break the cycle: removal of perineuronal nets in the lateral hypothalamus decreases cocaine relapse
}

\author{
Nathan J. Marchant ${ }^{1}$ \\ Neuropsychopharmacology (2019) 44:835-836; https://doi.org/10.1038/s41386-018-0245-Z
}

Cocaine addiction is responsible for several adverse health consequences, and the number of deaths attributable to cocaine has increased in the past years (National Center for Health Statistics, CDC Wonder). The major goal of treatment in cocaine addiction is relapse prevention. During cocaine use, cues in the environment (such as drug paraphernalia) become associated with the rewarding effect of cocaine. These memories persist during abstinence, and drug craving, now listed as a symptom of cocaine use disorder in DSM- 5 , can be triggered by exposure to these cues causing relapse [1]. To identify the brain regions critical for storing and expressing these cocaine memories, the cueinduced reinstatement of extinguished cocaine-seeking model is used in laboratory animals [2].

In their recent Neuropsychopharmacology article (https://doi. org/10.1038/s41386-018-0212-8), Blacktop and Sorg [3] focus on the role of a specialized form of the extracellular matrix (ECM) called perineuronal nets (PNNs), in the storage of cocaine memories in the brain. The PNNs are thought to constrain plasticity, because their formation co-occurs with the end of critical periods during the maturation of the cortex [4]. This function is achieved by PNNs by limiting the formation of new neuronal contacts, acting as scaffolding for molecules that inhibit synaptic formation, and constraining receptor mobility at the synapse [4]. A key factor in the chronically relapsing nature of addiction is the persistence of drug memories during abstinence. Because of the critical role of PNNs in the regulation of neuronal plasticity and memories, they have been proposed to be a potential target for intervention in drug addiction [5].

Blacktop and Sorg [3] focus on the role of PNNs in a small region of the lateral hypothalamic area (LHA), the anterior dorsal LHA (LHAad). Broadly, the LHA has been implicated with diverse functions, generally shown to be important for promoting motivated behavior [6]. While activity in LHA has been shown to be associated with context-induced reinstatement of cocaine seeking [7], to date there is no functional evidence demonstrating a critical role of LHA in promoting cue-induced reinstatement of drug seeking [2].

Blacktop and Sorg [3] used the well-established method of removing PNNs, intra-cranial infusion of chondroitinase $A B C$ (Ch$A B C)$, to test a role of this manipulation in cue-induced reinstatement of cocaine seeking. The authors injected $C h-A B C$ directly into LHAad, $16 \mathrm{~h}$ before the cue-induced reinstatement test. This manipulation, which was shown to substantially deplete the PNN in the LHAad, decreased cue-induced reinstatement. In a second experiment, the authors found no effect of removal of PNNs in LHAad on cue-induced reinstatement of extinguished sucrose seeking. Thus, the role of LHAad, and specifically the PNN within LHAad, appears to be critically important for the cocaine cue memories that drive reinstatement.

To further describe the anatomy and neurochemical makeup of the LHAad neurons that control cocaine seeking, the authors performed a series of immunohistochemical stains. The LHA is a well-known location of neurons that express several different peptides, such as orexin, melanin-concentrating hormone, galanin, and others [8]. While these peptides, and LHA in general, are implicated in motivated behaviors and drug seeking, Blacktop and Sorg [3] show that the LHAad comprises neurons that do not express any of these peptides. Rather, LHAad comprises a large population of parvalbumin (PV) expressing neurons and GABAexpressing neurons. Both PV and GABA neurons in LHAad are completely surrounded by PNNs. Thus, with the caveat that electrophysiological validation remains to be done, the population of neurons that are surrounded by PNNs in LHAad are likely GABAergic P-expressing neurons.

Finally, Blacktop and Sorg [3] placed the LHAad into the larger brain reward network, showing input from regions known to be important for promoting cue-induced reinstatement of cocaine seeking. Using anatomical tract tracing with retrobeads, they show that LHAad receives dense input from prelimbic PFC, a region that has previously been shown to be critical for cue-induced reinstatement of extinguished cocaine seeking [9]. Furthermore, the immunohistochemical stains revealed dense VGLUT2 expression in LHAad, suggesting that there are many glutamatergic terminals in LHAad. Outputs from LHAad were found to include both prelimbic PFC and the ventral tegmental area (VTA), while there was no evidence for projections from LHAad to the nucleus accumbens.

In summary, Blacktop and Sorg [3] have built on their previous work, where they showed that removal of LHAad PNNs decreases cocaine self-administration [10], to show that this region is also critical for relapse to cocaine seeking. These findings provide new evidence of a causal role of LHA in cue-induced reinstatement of cocaine seeking, and raise several interesting questions. From a circuitry perspective, this study shows anatomical connectivity between prelimbic PFC, LHAad, and VTA. This represents a likely circuit by which PFC can promote cue-induced reinstatement of cocaine seeking. Given the importance of dopamine in reinstatement of cocaine seeking [2], it will be of interest to show how activity in the VTA-projecting neurons in LHAad is changed by removal of PNNs. In conclusion, this study expands our understanding of LHA function, showing that the expression of cocaine cue memories is critically dependent on the expression of PNNs

${ }^{1}$ Department of Anatomy and Neuroscience, Amsterdam Neuroscience, VU University Medical Centre, Amsterdam, The Netherlands
Correspondence: Nathan J. Marchant (n.marchant@vumc.nl)

Received: 14 September 2018 Revised: 17 September 2018 Accepted: 20 September 2018

Published online: 24 October 2018 
Break the net, break the cycle: removal of perineuronal nets in the...

NJ Marchant

836

surrounding PV neurons in LHAad. These data provide evidence for a novel target for future studies examining how PNN removal changes the synaptic composition of this novel relapse circuit.

\section{FUNDING AND DISCLOSURE}

NJM was funded by a NWO VIDI grant (016.Vidi.188.022).

\section{REFERENCES}

1. O'Brien CP, Childress AR, McLellan AT, Ehrman R. Classical conditioning in drugdependent humans. Ann N Y Acad Sci. 1992;654:400-15.

2. Bossert JM, Marchant NJ, Calu DJ, Shaham Y. The reinstatement model of drug relapse: recent neurobiological findings, emerging research topics, and translational research. Psychopharmacology. 2013;229:453-76.

3. Blacktop JM, Sorg BA. Perineuronal nets in the lateral hypothalamus area regulate cue-induced reinstatement of cocaine-seeking behavior. Neuropsychopharmacology. 2018. https://doi.org/10.1038/s41386-018-0212-8.
4. Wang D, Fawcett J. The perineuronal net and the control of CNS plasticity. Cell Tissue Res. 2012;349:147-60.

5. Sorg BA, Berretta S, Blacktop JM, Fawcett JW, Kitagawa H, Kwok JC, et al. Casting a wide net: role of perineuronal nets in neural plasticity. J Neurosci. 2016;36:11459-68.

6. Marchant NJ, Millan EZ, McNally GP. The hypothalamus and the neurobiology of drug seeking. Cell Mol Life Sci. 2012;69:581-97.

7. Hamlin AS, Clemens KJ, McNally GP. Renewal of extinguished cocaine-seeking. Neuroscience. 2008;151:659-70.

8. Bonnavion P, Mickelsen LE, Fujita A, de Lecea L, Jackson AC. Hubs and spokes of the lateral hypothalamus: cell types, circuits and behaviour. J Physiol. 2016;594:6443-62.

9. McLaughlin J, See R. Selective inactivation of the dorsomedial prefrontal cortex and the basolateral amygdala attenuates conditioned-cued reinstatement of extinguished cocaine-seeking behavior in rats. Psychopharmacology. 2003;168:57-65.

10. Blacktop JM, Todd RP, Sorg BA. Role of perineuronal nets in the anterior dorsal lateral hypothalamic area in the acquisition of cocaine-induced conditioned place preference and self-administration. Neuropharmacology. 2017;118:124-36. 